\title{
Information Entropy Theory Based Recognition of the Validity of Contextual Information of Restaurants: An Empirical Study
}

\author{
Cong YIN, Liyi ZHANG, Meng TU, Xuan WEN, Yiran LI
}

\begin{abstract}
Contextual information plays a key role in personalized recommendations. However, not all contextual information plays a positive role in personalized recommendations. Therefore, it is critical to identify the effective contextual information to realize personalized recommendations. This study aims to develop a set of feasible context importance calculation methods that can identify effective contextual information in different application scenarios. The information entropy of each contextual dimension is calculated, and the validity of the context compared according to the magnitude of its entropy is determined based on the informational entropy theory. Subsequently, this approach is applied to hotel and catering service data to determine the valid context in the dining domain. The experimental results indicate that location, work-rest condition, weather, mood and companionship considerably influence consumers' behaviour and decisions in a catering environment, and the user preference in such contexts should be carefully considered.
\end{abstract}

Keywords: context; context validity; informational entropy, personalized recommendation; restaurant

\section{INTRODUCTION}

The rapid evolution of the mobile internet has accelerated its penetration into various areas of human society, which has facilitated the integration of online and offline resources and promoted the migration of ecommerce from computers to mobile phones. Thus, the mobile client is the new focus of e-commerce expansion [1]. Compared to the PC client, which is less affected by space and time, the interactive scenes of mobile shopping are more contextual, leading to their irreplaceable position as a new consumption form in social life. Although the integration of online and offline resources provides users with a variety of choices, it also inevitably leads to information overload. It is becoming increasingly difficult for customers to find information that matches their needs among the vast amount of information available regarding goods and services.

By using user-projected two-dimensional data mining, personalized recommendation has become an effective means to alleviate the information overload problem by mining user preferences and recommending information that meets their interests [2]. However, a user's personalized needs are more context-sensitive, the same user's preferences may change according to different contexts, and the user's preferences cannot be effectively mined by relying on the user-projected binary relationship alone [3]. Research regarding the importance of contextual information has been conducted by foreign scholars: Herlocker et al. suggested that recommendations should not be solely based on a user's historical feedback but also on other factors in the experience process [4]. Bettman discussed the decision-making mechanism in complex contexts and highlighted that a change in context could significantly impact the potential needs and final decisions of users [5]. Liu et al. [6] and Yu et al. [7] observed that different contextual factors influenced the potential needs of users. The importance of context to personalized recommendations was demonstrated in the studies of Gorgoglione et al. [8] and Mallat et al. [9]. It was considered that the efficiency and quality of personalized recommendations could be effectively improved by integrating the context factor into them. Domestic scholars have also performed relevant studies on the importance of contextual information in providing personalized recommendations: Zhuang et al. used a logistic regression model to analyse the user shopping contextual factors and noted that some contexts significantly impacted user purchasing decisions [10]. From the four dimensions of physical, social, behaviour and psychological context, Tu investigated the aspect of impulse shopping. The results indicated that these four dimensions exhibited significant positive effects on impulse buying, although the influence paths were different. Physical and behavioural contexts affected site purchases, while social and psychological contexts influenced unplanned purchases [11]. Hu and Cai suggested that integrating contextual factors into personalized recommendations could identify the preferences and requirements of different users more effectively [12]. Han $\mathrm{Li}$ indicated that context is a core factor that affects the user experience, particularly in the domain of mobile clients [13]. According to these studies, contextual information plays a key role in user behaviour and decision-making. This study exploits information entropy theory to identify effective contextual information in different catering environments to reduce the interference of invalid contexts.

\section{LITERATURE REVIEW}

No consensus regarding the definition of context exists in academic circles, although the definition provided by Dey et al. is widely accepted. The authors believed that "context refers to any information that can be used to represent the context in which an entity is located. The entity can be a person, place or object, whichever is relevant to the interaction between the user and the application, including the user and the application itself" [14]. In the field of context-aware recommendation, different scenarios involve different contexts and instances.

For instance, in information retrieval applications, context may involve interactive behaviours, retrieval tasks, keywords, themes, historical records and the social environment. [15]. In e-tourism applications, context may involve time, location, weather conditions, companions, 
traffic conditions, and visitor flow rates at tourist attractions. [16]. In the medical field, context may be related to gender, age, time, health status, and mood. [1718]. In e-commerce, the information can involve time, shopping intentions, seasons and internet conditions. [1921].

From the abovementioned scenarios, one can see that any application scene is associated with complicated contextual information. The common context types primarily include location, time, weather condition, mood, surrounding people, intention, activity status and social network. At the same time, the same context type can contain different context instances; for instance, time can be divided into seasons from spring to winter and by day from morning to night, and it can even be divided into minutes or seconds according to the specific time.

A wide range of contextual factors are involved in different application areas. Different types of contextual information have different influences on the quality of recommendations in the same application area. Some contextual factors have a considerable influence on the recommendation quality, while others have little or no effect. If certain less influential factors are incorporated into personalized recommendations, they will not effectively enhance the effect [22] but will increase the dimension and computational complexity of the recommendations; furthermore, such factors may even lead to a lower recommendation accuracy and user satisfaction, as they act as noise. Thus, the adoption of effective methods to select valid contexts is the premise of integrating the context of personalized recommendations.

In the existing studies, the introduction of contextual factors depends more on the researchers' understanding of the application field, for instance, the choice of "location" as a contextual factor to recommend the nearest cinema to a user or "mood" to match music to a user's current mood. This method of selection of common sense based contextual factors is simple and easy to understand. However, this method is subjective and lacks theoretical support, and therefore, it often leads to inadequate considerations of and missing key contexts. Liu et al. indicated that the selection of contextual factors belongs to the category of feature selection, and the featured information of the specific application should be specified by domain experts [22]. However, the specific solution is not clearly reported in the literature. Some scholars have used mathematical statistics or machine learning methods to determine the influence of different contextual information on different applications or services, which corresponds to the context validity. Van et al. suggested that the input rules for users could be provided by the system and that users could input the information corresponding to their interest according to the rules. Although this method is objective, comprehensive, authentic and reliable, very few users are willing to spend time to provide their preference context to the system through a human-computer interaction, and the real-time performance is poor. Baltrunas et al. performed a contextual correlation assessment to determine the impact of certain contextual conditions on users' ratings in tourism [23]. This assessment was performed by asking users to imagine a given contextual condition and evaluate its impact; however, Ono et al. questioned this approach because users' ratings in real and hypothetical contexts are different [24]. Odić et al. used the paired t-test to detect the valid contextual information in a dataset [25]. However, in the adopted dataset, all the context types were absolute, and thus, the paired t-test was not applicable. Liu et al. used the $\chi^{2}$ test to detect the relevant context [26]; however, this method is not suitable for small datasets, such as a new system and those involving the cold start problem.

In conclusion, related research on context validity detection remains limited. This study incorporates the informational entropy theory to help with detection. The information entropy of the contextual instance is calculated according to the positive feedback of different categories of items selected by a user under different contextual instances, and the information entropy of the entire contextual dimension is calculated considering the conditional entropy to reflect the confusion degree of the user choosing an item in the context. A larger contextual information entropy corresponds to a lower context validity, and a smaller entropy corresponds to a smaller context validity.

\section{RESEARCH THEORIES AND METHODS}

\subsection{Theoretical Foundation}

Information is the knowledge source for human beings to understand and transform the world. Information is a description of uncertainty, and thus, its determination is necessary. Shannon proposed that informational entropy can be used to measure the uncertainty of random variables in a system. A larger system informational entropy indicates more chaotic information [27]. The informational entropy theory can be described as follows:

Assume an off-line random variable; its probability distribution is

$$
\left[\begin{array}{c}
X \\
P(x)
\end{array}\right]=\left[\begin{array}{cccc}
x_{1} & x_{2} & \cdots & x_{n} \\
p_{1} & p_{2} & \cdots & p_{n}
\end{array}\right] \text { and } 0 \leq p_{i} \leq 1, \sum_{\mathrm{i}=1}^{n} p_{i}=1
$$

Subsequently, the self-information quantity of any random event $I\left(x_{i}\right)$ is a negative value of the logarithm of the occurrence probability of the random event, that is,

$$
I\left(x_{i}\right)=\log \left(p_{i}\right)
$$

The self-information quantity $I\left(x_{i}\right)$ is contained in the information $x_{i}$ sent by source $X$. The message itself determines the amount of information. Shannon defines information entropy using the average self-information quantity, which is defined as

$$
\begin{aligned}
& H(X)=H\left(p_{1}, p_{2}, \ldots, p_{n}\right)= \\
& =\sum_{i=1}^{n} p_{i} \cdot I\left(x_{i}\right)=-\sum_{i=1}^{n} p_{i} \cdot \log p_{i}
\end{aligned}
$$

A larger uncertainty of the variables corresponds to a larger entropy; if the distribution of the variables is more orderly, the entropy is smaller. Therefore, information entropy is an important measure of the information order in a source. 


\subsection{Conceptual Framework}

The conceptual framework is shown in Fig. 1. This study analyses the contextual information that is involved in specific scenarios based on the obtained user and item data and introduces the informational entropy theory into the process of contextual validity detection to calculate the entropy of these contextual pieces of information. A larger contextual information entropy corresponds to a lower validity; conversely, a smaller entropy value corresponds to a higher contextual validity. Subsequently, the context importance degree is calculated according to the contextual dimension corresponding to information entropy. A higher degree corresponds to a higher importance of the context, which indicates that in the current application scenario, the impact of this context on the user's behaviour and decisionmaking is greater, and thus, the contextual dimension is more valid. The specific research methods are as follows:

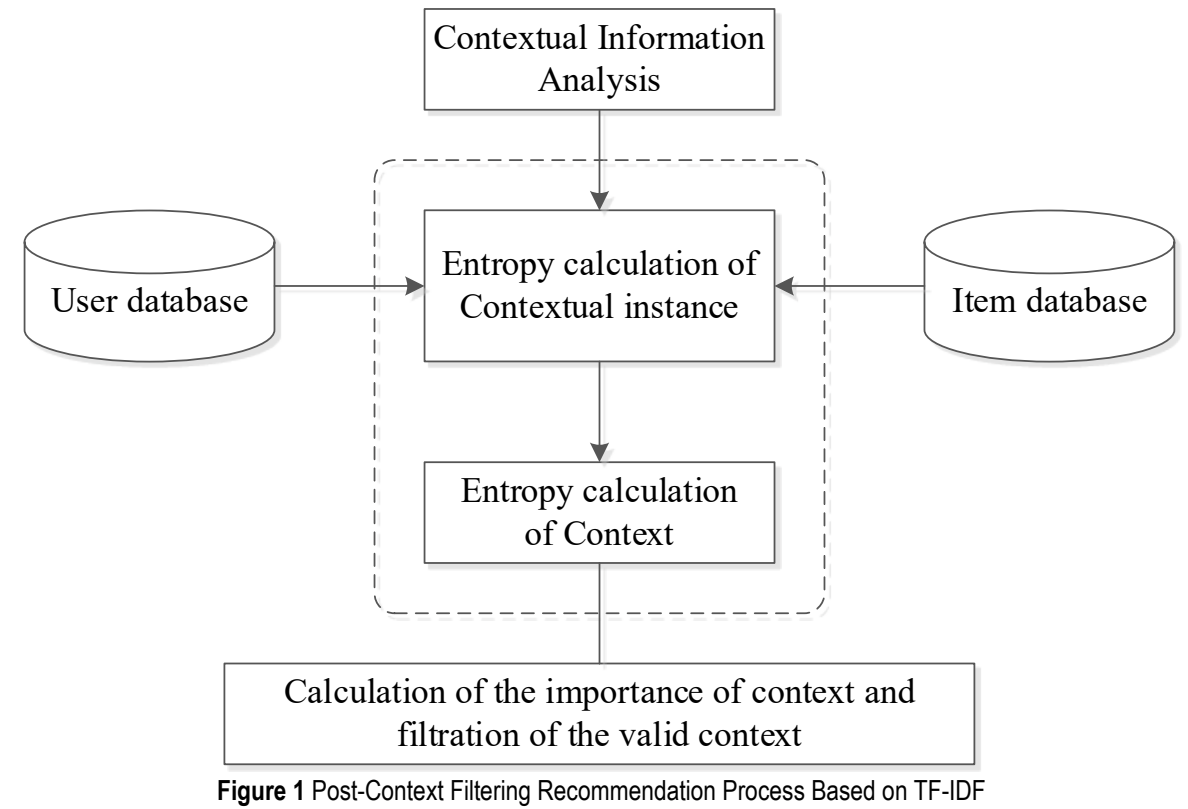

\subsection{Research Methodology}

Contextual information entropy refers to the entropy value of different items selected by a user under a certain context. Calculation of the contextual information entropy involves calculating the information entropy according to the positive feedback of different categories of items selected by the user under different contextual instances. The information entropy of the entire contextual dimension is calculated using the conditional entropy, and this process can be divided into the following steps:

\subsubsection{Calculation of the User's Positive Feedback Probability Distribution for Different Item Categories in Each Case}

A positive feedback is the user's preference for an item, reflected in an implicit or explicit manner, whereas the user's dislike corresponds to a negative feedback. For the explicitly rated individualized recommendation, the user's feedback expresses the degree of preference for the item through a specific score, which is often based on a five-point system: less than three points indicate dislike, and three points and above indicate that the user likes the item. In recommendations with implicit feedback, the user's feedback represents preferences for items through the user's behaviour (collection, shopping cart, purchase, etc.). Quantization of the preference is usually expressed as 0 and 1 , where 1 indicates like and 0 indicates dislike. This feedback information is helpful to analyse the user's preferences in different context cases. Therefore, influenced by the contextual instance $C_{m k}$, the positive feedback probability of the items under category $V_{i}$ is as follows:

$$
p_{C_{m k}}\left(V_{i}\right)=\frac{\operatorname{count}\left\{u_{i}=1 \mid V_{i}, C=C_{m k}\right\}}{\operatorname{count}\left\{u_{i}=1 \mid C=C_{m k}\right\}}
$$

where $u_{i}=1$ indicates a positive feedback from the user and count $\left\{u_{i}=1 \mid V_{i}, C=C_{m k}\right\}$ represents the user's positive feedback number for an item under category $V_{i}$ in the context $C_{m k}$. count $\left\{u_{i}=1 \mid C=C_{m k}\right\}$ represents the user's positive feedback number for all the items in the context $C_{m k}$. Based on formula (3), the positive probability set of the user for items from different categories in the contextual instance $C_{m k}$ is as follows:

$$
p_{C_{m k}}(V)=\left\{p_{C_{m k}}\left(V_{1}\right), \ldots, p_{C_{m k}}\left(V_{i}\right), \ldots, p_{C_{m k}}\left(V_{n}\right)\right\}
$$

where $p_{C_{m k}}(V)$ represents the confusion degree of the user's choice of different categories of items under the contextual instance $C_{m k}$. When the values of the elements in a set are closer to each other, the user's preference for different categories of items under the contextual instance $C_{m k}$ does not have any significant difference. By contrast, if the values of the elements are considerably different, the user has a comparative preference for a particular category of items in the contextual instance $C_{m k}$. 


\subsubsection{Calculation of the Information Entropy of a Contextual Instance}

According to formula (4), the entropy value of the items selected by the user for all categories under the contextual instance $C_{m k}$ can be calculated as follows:

$$
I\left(C_{m k}\right)=-\sum_{i=1}^{n} p_{C_{m k}}\left(V_{i}\right) \cdot \log _{n} p_{C_{m k}}\left(V_{i}\right)
$$

where $n$ is the number of item categories. $I\left(C_{m k}\right)$ indicates the confusion degree with which users select different categories of items under the contextual instance $C_{m k}$. A larger entropy value means that a user is more confused in regard to choosing different categories of items in the case, that is, the user exhibits no clear preference for any category of items for a given case.

\subsubsection{Calculation of Contextual Information Entropy}

Because different contextual factors involve different context case distributions, contextual information entropy can be calculated considering conditional entropy according to the probability of the context case distribution $p\left(C_{m k}\right)$ and its information entropy $I\left(C_{m k}\right)$. The calculation formula is as follows:

$$
E\left(C_{m}\right)=\sum_{k=1}^{t} p\left(C_{m k}\right) \cdot I\left(C_{m k}\right)
$$

where $t$ is the contextual instance number included in the contextual factor $C_{m} ; 1 \leq k \leq t$.

According to the information entropy connotation, when the entropy value of the contextual factor $C_{m}$ is greater, the selection of items in different categories under the contextual case is more likely to have equal occurrence probabilities. That is, the user's preference is more balanced for items in different categories under the contextual case. This aspect indicates that the user has little influence on item selection in this context. When the item selection probability is $1 / n$ under the case condition, the contextual information entropy is maximum, that is, $I\left(C_{m k}\right)$ $=\log _{n} n=1$, and the contextual factor has no influence on item selection. Conversely, when the entropy value of the contextual factor $C_{m}$ is smaller, the user has a clear preference among items with different attributes under the current context $C_{m}$. That is, under the influence of the contextual factor, the user exhibits a preference for items with different attributes. When a user chooses an item with a certain attribute, the positive feedback probability distribution of the user's selection of items with different attributes is 0 and 1 ; at this point, $\log _{n} 1=0$ or $\log _{n} 0=-\infty$, and the contextual information entropy is 0 . A smaller information entropy corresponds to a greater impact of context on the current decision, whereas a greater information entropy corresponds to a smaller impact. In other words, the information entropy of the context is inversely proportional to the impact degree of the user's decision.

Formula (7) can be used to measure the influence of the contextual information in a specific scene; a larger $I\left(C_{m}\right)$ indicates a greater influence, and a smaller $I\left(C_{m}\right)$ corresponds to a smaller influence.

$$
I\left(C_{m}\right)=1-E\left(C_{m}\right)
$$

\section{EMPIRICAL ANALYSIS \\ 4.1 Experimental Object Selection}

Food is a basic necessity of people, and foods and beverages are closely related with the daily life of people. In particular, currently, with the increasing pace of life, people choose to eat out more often. As a high frequency consumer industry, the consumption situation of the catering industry is extremely contextual, and at different times and locations a user's consumption intentions are different. Therefore, in this study, the catering industry of Dianping.com is considered as the research object, and the consumer consumption score data from the Wuhan area is analysed.

\subsection{Data Acquisition and Pre-Processing}

Crawler software was used to capture users' consumption evaluation information and corresponding store information for Dianping, including users' ID, shop ID, consumption time, shop name, store catering category, rating and evaluation information. Since the number of users was large, the evaluation was not supervised and the reliability could not be completely guaranteed; therefore, malicious user evaluations were deleted. A total of 132 Wuhan users and 60,142 reviews were obtained, considering 3,295 stores. The related contextual information was obtained as follows:

Location context. The locations of the restaurant could be determined considering the store locations, and according to the Wuhan city centre zoning, they were divided into Wuchang, Hongshan, Qingshan, Hanyang, Jiang'an, Jianghan, and Qiaokou.

Interactive context. If the users consumed food in the store, they could be divided into new or returning customers. According to the users' historical consumption records and reviews, keywords such as "first time", "three or four times", "frequently visit", "multiple consumption", "several visits" and other relevant words were extracted.

Week context. This value could be obtained according to the consumption date directly related to the specific week.

Work/Rest context. This context included a user's working day, rest day and holiday. The specific types of work or rest on the consumption day could be determined by the relevant regulations of the general office of the Chinese State Council.

Season context. The season context is the season in which the user consumed food. However, the common division of the four seasons neglects regional differences. Thus, this study adopted the "climate-temperature" method proposed by domestic scholar Baokun Zhang to take the average temperature of five days as one small element; the details are shown in Fig. 2. According to this method, the distribution characteristics of the four seasons of short spring and autumn and long summer and winter could be effectively reflected in Wuhan. 
Decision context. The decision context is the user's current consumption decision, which is decided by the user independently or recommended by others and can be extracted according to some keywords from the users' comments, such as "friend recommended" and "best friend recommendation".

Companion context. This context includes contextual instances such as dining alone or with friends, partners or family members. The value could be obtained according to keywords such as "one person", "friend", "best friend", "buddy", "girlfriend", "husband", "parent", "child", "father" and so on.

Temperature context. The climate of the current consumption date can be divided into three categories according to the maximum temperature, minimum temperature and average temperature, which can be further divided into three categories: hot, moderate and cold.

Weather context. This context corresponds to the weather of the current consumption date, for instance, overcast, sunny, rainy, snowy, or cloudy conditions.

Mood context. This context consists of the mood state of the user in the process of consumption, such as positive, negative and neutral. This study used the semantic polarity analysis method proposed by Pan and Lin to analyse the users' comment information and obtain the corresponding mood context [28].

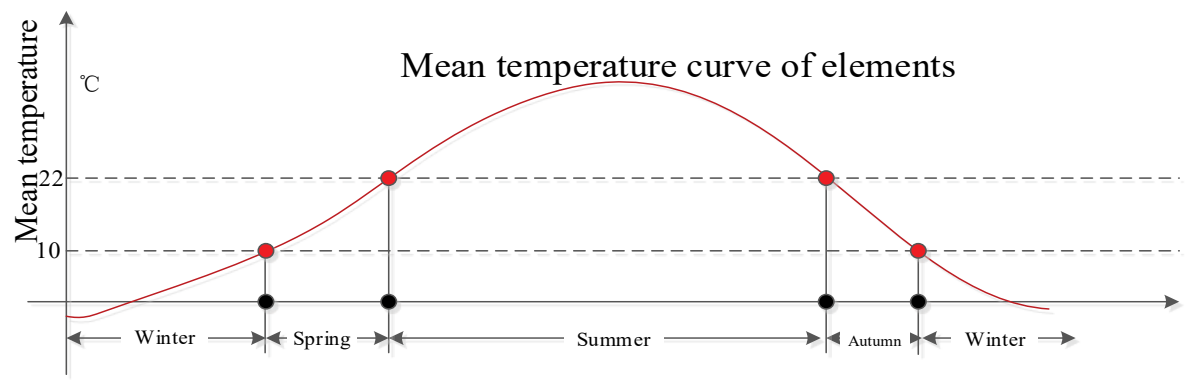

Figure 2 Schematic diagram of season division according to the "climate-temperature" method

The types of contexts involved in the catering application scenarios and their instances are presented in Tab. 1.

Table 1 Context types and instances in the restaurant application scene

\begin{tabular}{|l|l|}
\hline \multicolumn{1}{|c|}{ Dimension } & \multicolumn{1}{c|}{ Instance } \\
\hline Location & $\begin{array}{l}\text { Wuchang, Hongshan, Qingshan, Hanyang, Jiang'an, } \\
\text { Jianghan, Qiaokou. }\end{array}$ \\
\hline Interaction & New customer, Returning customer \\
\hline Week & $\begin{array}{l}\text { Monday, Tuesday, Wednesday, Thursday, Friday, } \\
\text { Saturday, Sunday }\end{array}$ \\
\hline Work/Rest & Working day, Rest day, Holiday \\
\hline Season & Spring, Summer, Autumn, Winter \\
\hline Decision & Independent, Recommended \\
\hline Companion & Alone, with Friend, with Partner, with Family \\
\hline Temperature & Hot, Moderate, Cold \\
\hline Weather & Overcast, Sunny, Rain, Snow, Cloudy \\
\hline Mood & Positive, Negative, Neutral \\
\hline
\end{tabular}

\subsection{Calculation and Analysis of Contextual Information Entropy}

Combining the user and item score data, the information entropy and context importance of the abovementioned contextual information were calculated, as shown in Tab. 2 .

Table 2 Calculated information entropy in the restaurant application scene

\begin{tabular}{|l|c|c|}
\hline $\begin{array}{c}\text { Contextual } \\
\text { Dimension }\end{array}$ & Information Entropy & Context Importance \\
\hline Decision & 0.9878 & 0.0122 \\
\hline Location & 0.6845 & 0.3155 \\
\hline Week & 0.9988 & 0.0012 \\
\hline Work/Rest & 0.6576 & 0.3424 \\
\hline Season & 0.9437 & 0.0563 \\
\hline Weather & 0.6559 & 0.3441 \\
\hline Temperature & 0.9798 & 0.0202 \\
\hline Companion & 0.7335 & 0.2665 \\
\hline Mood & 0.7844 & 0.2156 \\
\hline Interaction & 0.9348 & 0.0652 \\
\hline
\end{tabular}

To make the information more intuitive, Tab. 2 is plotted as a histogram, as shown in Fig. 3.

From this table, it can be seen that the contextual influence of the decision (0.0122), week (0.0012), season $(0.0563)$, temperature $(0.0202)$ and interaction $(0.0652)$ is less than 0.1 , indicating that these information points have little effect on the user preference in catering $\mathrm{O} 2 \mathrm{O}$. Similarly, the contextual influence of the location (0.3155), work/rest (0.3424), weather (0.3441), companion (0.2665) and mood $(0.2156)$ is comparatively higher, indicating that these information points exert considerable influence.

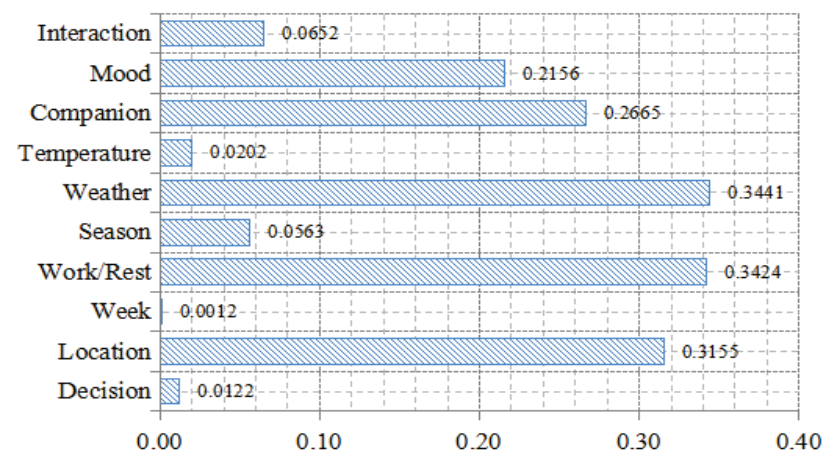

Figure 3 Calculated information entropy in the restaurant application scene

\subsubsection{Optimal Weight Coefficient Determination}

During the initial prediction scoring, the traditional score similarity and item category similarity are weighted. The weight coefficient is closely related to the accuracy of the prediction score. In this study, the weight coefficient $\beta$ increases from 0.1 to 0.9 in intervals of 0.1 , the nearest neighbour number increases from 10 to 60 in intervals of 10 , and the corresponding MAE value is calculated, as shown in Fig. 4. It can be seen from the graph that the 
average absolute error MAE value is the smallest, that is, the best weight coefficient occurs when $\beta=0.6$.

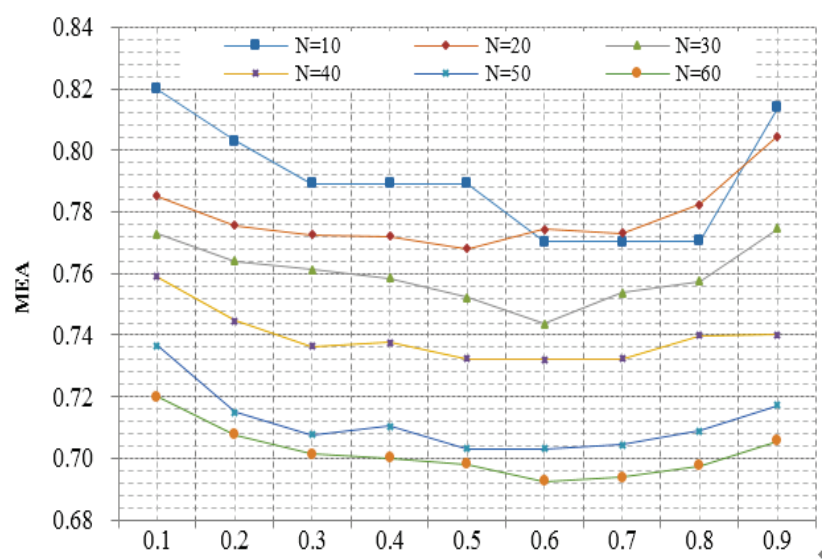

Figure 4 MAE values for different $\beta$ values and neighbouring numbers

For a high-frequency consumption industry, the week, as a regular time context, has a significant impact on consumer decision-making. The season and temperature contexts are likely to have more considerable impacts on users' preferences in some specific catering industries. For example, crayfish is a popular food in Wuhan during each summer, while crayfish restaurants are packed and closed in other seasons. When the temperature is low, customers are more likely to choose hot pot, Sichuan or Hunan cuisine. However, for the entire catering industry, most restaurants are less affected by season or temperature. Although the importance of the companion and mood contexts is lower than that of the location, work/rest and weather, its importance is considerably higher than that of the decision making, week, season, temperature and interaction aspects. This finding indicates that these contexts play a key role in the decision making of catering $\mathrm{O} 2 \mathrm{O}$ consumption.

The decision and interaction are two important contexts in the decision-making stage of the interaction between users and items. The calculation of the information entropy indicates that the influence of these aspects is relatively small. The in-depth analysis indicates that the main reason for this result is likely that the information was obtained from users' comments. However, it is inevitable that some deviations exist due to the lack of this type of contextual information. Therefore, the acquisition needs to be strengthened in future research.

In summary, the information entropy calculation indicates that the context types that affect users' preference in catering $\mathrm{O} 2 \mathrm{O}$ are mainly related to five types: location, work/rest, weather, mood and companion.

\section{CONCLUSIONS}

Based on an in-depth analysis of the importance of contextual information, this study uses the informational entropy theory to improve context validity detection; however, this aspect requires further investigation. First, most of the contextual information in this study depends on keyword extraction from users' comments, which are not present in some contexts. This aspect results in a lack of contextual information, which affect the calculation of the context validity. A more intelligent and effective method for obtaining the contextual information needs to be investigated in depth. Second, the data sample selection needs to be enriched. In this study, catering review data of only 132 Wuhan users were obtained, although Wuhan has tens of thousands of users. To identify the valid context more effectively, follow-up research needs to be strengthened in regard to these methods.

\section{Acknowledgment}

This work is supported by the National Natural Science Foundation of China (71874126) the Scientific Research Foundation of Chongqing University of Technology.

\section{REFERENCES}

[1] iResearch.com. (2016). China O2O industry development report simplified version. Shanghai.

[2] Borchers, A., Herlocker, J., Konstan, J., \& Reidl, J. (1998). Ganging up on information overload. Computer, 31(4), 106108. https://doi.org/10.1109/2.666847

[3] Chen A. (2005) Context-Aware Collaborative Filtering System: Predicting the User's Preference in the Ubiquitous Computing Environment. In: Strang T., Linnhoff-Popien C. (eds) Location- and Context-Awareness. LoCA 2005. Lecture Notes in Computer Science, vol. 3479. Springer, Berlin, Heidelberg, 244-253. https://doi.org/10.1007/11426646_23

[4] Herlocker, J. L., Konstan, J. A., Borchers, A., \& Riedl, J. (1999). An algorithmic framework for performing collaborative filtering. SIGIR 99: International ACM SIGIR Conference on Research \& Development in Information Retrieval (DBLP), August 15-19, Berkeley, CA, USA. 1999:230-237. https://doi.org/10.1145/312624.312682

[5] Bettman, J. R., Luce, M. F., \& Payne, J. W. (1998). Constructive consumer choice processes. Journal of Consumer Research, 25(3), 187-217. https://doi.org/10.1086/209535

[6] Liu, Q. H., Zhang, X. Y., Zhang, L. Y., \& Zhao, Y. (2019). The interaction effects of information cascades, word of mouth and recommendation systems on online reading behavior: An empirical investigation. Electronic Commerce Research, 19(3), 521-547. https://doi.org/10.1007/s10660-018-9312-0

[7] Yu, Z. (2006). Supporting context-aware media recommendations for smart phones. Pervasive Computing, 5(3), 68-75. https://doi.org/10.1109/MPRV.2006.61

[8] Gorgoglione, M., Palmisano, C., \& Tuzhilin, A. (2006). Personalization in Context: Does Context Matter When Building Personalized Customer Models? Proceedings of the $6^{\text {th }}$ IEEE International Conference on Data Mining (ICDM 2006), 18-22 December, Hong Kong, China. IEEE, 222-231.

[9] Mallat, N., Rossi, M., Tuunainen, V. K., \& Öörni, A. (2009). The impact of use context on mobile services acceptance: the case of mobile ticketing. Information \& Management, 46(3), 190-195. https://doi.org/10.1016/j.im.2008.11.008

[10] Zhuang Gui-jun, Zhou Nan, \& Li Fu-an. (2004) Impact of Situational Factors on Shoppers' Buying Decision: An Initial Study. Journal of Applied Statistics and Management, 23(4), 7-13.

[11] Tu Li. (2010). The Influence of Situational Factors on Impulse Buying Behavior. Dissertation, Hubei University.

[12] Mu, H., Hu, S., \& Cai, Q. (2010). Personalized recommendation method on loosely coupled situation with Extended Research. Library and Information Service, 371376. 
[13] Han Li. (2014). The Current State of Context-aware Research. Computer and Information Technology, 22(6), 45.

[14] Abowd, G. D., Dey, A. K., Brown, P. J., Davies, N., Smith, M., \& Steggles, P. (1999). Towards a better understanding of context and context-awareness. Handheld and ubiquitous computing. Springer Berlin Heidelberg, 304-307. https://doi.org/10.1007/3-540-48157-5 29

[15] Yan Ye, Yang Zhao, Jennifer Shang, \& Liyi Zhang. (2019). A hybrid IT framework for identifying high-quality physicians using big data analytics. International Journal of Information Management, 47(4), 65-75. https://doi.org/10.1016/j.jijnfomgt.2019.01.005

[16] Carolis, B. D., Mazzotta, I., Novielli, N., \& Silvestri, V. (2009). Using common sense in providing personalized recommendations in the tourism domain. In Proceedings of the Workshop on Context-Aware Recommender Systems.

[17] Yang Zhao, Kunlin Li, \& Liyi Zhang. (2019). A metaanalysis of online health adoption and the moderating effect of economic development. International Journal of Medical Informatics, 127(7), 68-79. https://doi.org/10.1016/j.ijmedinf.2019.04.015

[18] Yang Zhao, Qi Ni, \& Ruoxin Zhou. (2018). What Factors Influence the Mobile Health Service Adoption? A Metaanalysis and the Moderating Role of Age. International Journal of Information Management, 43(6), 342-350. https://doi.org/10.1016/j.jijinfomgt.2017.08.006

[19] Daomeng Guo, Yang Zhao, Liyi Zhang, Xuan Wen, \& Cong Yin. (2019). Conformity Feedback in an Online Review Helpfulness Evaluation Task Leads to Less Negative Feedback-Related Negativity Amplitudes and More Positive P300 Amplitudes. Journal of Neuroscience, Psychology, and Economics. https://doi.org/10.1037/npe0000102

[20] Oku, K., Nakajima, S., Miyazaki, J., \& Uemura, S. (2006). Context-Aware SVM for Context-Dependent Information Recommendation. IEEE Computer Society. 109-112. https://doi.org/10.1109/MDM.2006.56

[21] Liu, Q. H., Huang, S., \& Zhang, L.Y. (2016). The influence of information cascades on online purchase behaviors of search and experience products. Electronic Commerce Research, 16(4), 553-580. https://doi.org/10.1007/s10660-016-9220-0

[22] Qihua Liu, Xiaoyu Zhang, Shan Huang, Liyi Zhang, \& Yang Zhao (2020). Exploring Consumers' Buying Behavior in a Large Online Promotion Activity: The Role of Psychological Distance and Involvement. Journal of theoretical and applied electronic commerce research, 15(1), 1-15. https://doi.org/10.4067/S0718-18762020000100106

[23] Baltrunas, L., Ludwig, B., Peer, S., \& Ricci, F. (2012). Context relevance assessment and exploitation in mobile recommender systems. Personal and Ubiquitous Computing, 16(5), 507-526. https://doi.org/10.1007/s00779-011-0417-x

[24] Ono, C., Takishima, Y., Motomura, Y., \& Asoh, H. (2009). Context-Aware Preference Model Based on a Study of Difference between Real and Supposed Situation Data. Proceedings of the $17^{\text {th }}$ International Conference User Modeling, Adaptation, and Personalization (UMAP 2009), formerly UM and AH, Trento, Italy, June 22-26, SpringerVerlag, 102-113. https://doi.org/10.1007/978-3-642-02247-0_12

[25] Odic, A., Tkalcic, M., Tasic, J. F., \& Kosir, A. (2013). Predicting and detecting the relevant contextual information in a movie-recommender system. Interacting with Computers, 25(1), 74-90. https://doi.org/10.1093/iwc/iws003

[26] Liu, L., Lecue, F., Mehandjiev, N., \& Xu, L. (2010). Using Context Similarity for Service Recommendation. Semantic Computing (ICSC), 2010 IEEE Fourth International Conference on. IEEE, 277-284

https://doi.org/10.1109/ICSC.2010.39
[27] Shannon, C. E. (1948). A mathematical theory of communication. Bell Labs Technical Journal, 27(4), 379423. https://doi.org/10.1002/j.1538-7305.1948.tb01338.x

[28] Yu, P. \& Hong-Fei, L. (2008). Restaurant reviews mining based on semantic polarity analysis. Computer Engineering, 34(17), 208-210.

\section{Contact information:}

Cong YIN, PhD

Chongqing Intellectual Property School,

Chongqing University of Technology,

No. 69 Hongguang Avenue, Banan District, Chongqing, China

cyin@cqut.edu.cn

\section{Liyi ZHANG, Professor}

(Corresponding author)

School of Information Management,

Wuhan University,

No. 299 Bayi Road, Wuchang District

Wuhan City, Hubei Province, China

lyzhang@whu.edu.cn

\section{Meng TU, PhD}

Chongqing Intellectual Property School,

Chongqing University of Technology,

No. 69 Hongguang Avenue, Banan District, Chongqing, China

amytwe0214@hotmail.com

\section{Xuan WEN, PhD}

School of Information Management

Wuhan University,

No. 299 Bayi Road, Wuchang District, Wuhan City, Hubei Province, China

Xuan_W113@163.com

\section{Yiran LI, PhD}

School of Information Management,

Wuhan University,

No. 299 Bayi Road, Wuchang District

Wuhan City, Hubei Province, China

yiran.li@whu.edu 